\title{
Proposta de Arquitetura Utilizando o Paradigma SOA para o Avatar Educação
}

\author{
Title: Proposed Architecture Using the SOA Paradigm for Education Avatar
}

\author{
Marcos de Souza Oliveira \\ Escola Politécnica de Pernambuco \\ Universidade de Pernambuco \\ 50.720-001 - Recife, Brasil \\ Marcosd3souza@gmail.com
}

\author{
Alexandre Magno Andrade Maciel \\ Escola Politécnica de Pernambuco \\ Universidade de Pernambuco \\ 50.720-001 - Recife, Brasil \\ amam@ecomp.poli.br
}

Resumo A demanda por cursos de ensino à distância vem alcançando números significativos no Brasil, com isso cresce também a atenção nos ambientes virtuais de aprendizado, que são responsáveis por ministrar esses cursos. A interação nesses ambientes é fundamental para que os objetivos do curso sejam alcançados. Neste sentido o uso de assistentes virtuais, como o Avatar Educação, facilitam a comunicação e interação nesses ambientes. Apesar do uso de tecnologias multiplataformas, o Avatar Educação foi desenvolvido especificamente para o ambiente Moodle. Assim este trabalho propõe uma nova arquitetura, fazendo uso do paradigma de arquitetura orientada a serviço, para tornar o funcionamento deste assistente mais independente do ambiente Moodle.

Palavras-Chave: Moodle, Avatar Educação, SOA, Ambientes Virtuais de Aprendizagem

\begin{abstract}
The demand for distance education courses has achieved significant numbers in Brazil, it is also growing attention in virtual learning environments (VLE), which are responsible for providing these courses. The interaction in these environments is fundamental to that the course goals are achieved. Thus the uses of virtual assistants, such as Education Avatar, facilitate the communication and interaction these environments. Although use of multiplatforms technologies the Education Avatar was developed in Moodle environment. So this work proposes the new architecture, using service-oriented architecture paradigm, making the operation from assistant more independent of Moodle environment.
\end{abstract}

Keywords: Moodle, Avatar Education, SOA, Virtual Learning Environment 


\section{Introdução}

O ensino a distância (EAD) no Brasil vem obtendo um crescimento acelerado. $\mathrm{Na}$ análise do censo do ensino superior de 2014, fornecido pelo instituto nacional de ensino e pesquisa (INEP), a modalidade teve uma participação de $17,1 \%$ no total de matrículas e um crescimento de $16,3 \%$ no período de $2013-2014$, superior a modalidade presencial $(5,4 \%)$ [16]. Este cenário é reflexo das possibilidades oferecidas pelo uso de TIC's em educação[1]. Nesse contexto, os ambientes virtuais de aprendizagem (AVAs) tem se tornado essenciais tanto no meio acadêmico quanto no ambiente corporativo. No meio acadêmico é utilizado para cursos de graduação, extensão e pós-graduação. No meio corporativo é utilizado, por exemplo, para prover cursos de treinamentos para funcionários sobre determinado produto, ou serviço, oferecido pela organização. Para dar suporte a realização desses cursos, os AVAs fornecem várias ferramentas como locais para armazenamento e disponibilização de materiais de aula, fóruns, chats, avaliações, entre outras [2].

Segundo Litto e Formiga [1] um dos grandes desafios da modalidade de ensino a distância é conseguir atingir um equilíbrio entre um estudo independente e as atividades interativas. Um exemplo de programa que não possui atividades interativas são cursos de educação bancária, que aplicam um método antidialógico. $\mathrm{Na}$ abordagem os alunos devem apenas receber, repetir, memorizar, guardar e arquivar conteúdos. Para que haja um estudo independente envolvendo atividades interativas em um AVA pode se ter, por exemplo, uma interatividade refletida, na qual as respostas de outras pessoas, incluindo livros e especialistas, são mostradas para que o aluno possa refletir e formular suas próprias respostas sobre um determinado tópico de discussão [1].

Devido a sua importância no processo de ensino e aprendizagem, em cursos a distância, diversas formas de interatividade foram surgindo. A proposta inicial foi de Moore (1989), que observou a relação entre os atores de um curso EAD, surgindo assim três tipos de interação: aluno/professor, aluno/aluno e aluno/conteúdo. Posteriormente os AVA's também foram considerados como atores no processo de ensino/aprendizagem [1].

Diante desse cenário, em Maciel et al. (2014), foi proposto o uso de um assistente virtual integrado ao AVA Moodle para dar suporte a aprendizagem online, visando promover uma melhor interação e comunicação entre os alunos e o ambiente. Nos experimentos realizados deste estudo, verificou-se que o assistente foi utilizado com mais frequência no início de um curso, devido a necessidade de adaptação do aluno com as ferramentas oferecidas pelo ambiente.
A arquitetura estabelecida para viabilizar o desenvolvimento do assistente utilizou o middleware Avatar Vocálico desenvolvido por Rodrigues et al. (2012) e o framework FIVE desenvolvido por Maciel et al. (2010). Apesar dos bons resultados obtidos, o desenvolvimento do plugin foi realizado atendendo especificamente a estrutura do ambiente Moodle.

Desse modo, o objetivo geral deste trabalho é realizar a refatoração da atual arquitetura, utilizando as boas práticas oferecidas pelo paradigma de arquiteturas orientadas a serviço. Para isto, a Seção 2 irá abordar os aspectos teóricos que viabilizam a proposta da arquitetura, a Seção 3 irá apresenta a arquitetura proposta, a Seção 4 irá avaliar a viabilidade da solução fornecida e por fim a Seção 5 irá abordar as conclusões obtidas neste trabalho.

\section{Fundamentação Teórica}

\subsection{Avatar Educação}

No Brasil há uma grande expansão do uso de plataformas de ensino à distância (EAD). Essa demanda tornou-se possível com o advento da Web 2.0, também conhecida como web social. Neste modelo é observado uma maior interação e colaboração entre os usuários. É possível haver conversações, diálogos e postagens de opiniões por parte desses usuários. Esses ambientes também utilizam diversas ferramentas que auxiliam os tutores no acompanhamento de suas aulas nos cursos EAD, tais ferramentas são chats, fóruns, vídeo-aulas, etc.[15]

Visando promover a comunicação e auxiliar ainda mais os tutores no acompanhamento de suas aulas, em Maciel (2014) foi desenvolvido um assistente virtual, chamado Plugin Avatar de Educação, que reproduz mensagens com voz sintética para repassar avisos deixados pelo tutor ou quando há a inserção de um novo conteúdo no ambiente. O Avatar ao fazer uso de expressões faciais, que demonstram sentimentos de afeto e se assemelham aos sentimentos humanos facilita a comunicação. Segundo Prola e Viccari (2003) esses personagens aumentam a afetividade das aplicações de educação.

Na Figura 1 é possível ver o design do Avatar com suas duas opções de interação, o botão mensagem e o botão de notificação. O funcionamento do Avatar para a opção "Notificação" parte da realização de consultas predefinidas na base de dados do ambiente Moodle. Caso esta consulta retorne novos materiais ou atividades adicionados após o último login do aluno ou atividades pendentes, será gerada uma mensagem padrão notificando sobre a situação do aluno perante esses materiais ou atividades, em seguida essa mensagem, no formato textual, será encaminhada para o processo de síntese de voz para a geração do áudio a ser reproduzido pelo Avatar. 


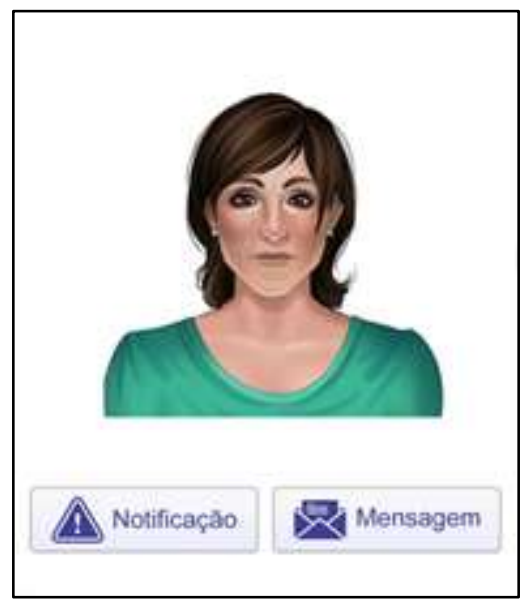

Figura 1: Plugin no Moodle do Avatar Educação. Maciel (2014).

Quando houver a ação na opção "Mensagem" apenas haverá a reprodução da animação, pois o tutor ao registrar uma mensagem, através da opção de edição do plugin, o processo de síntese de voz será realizado automaticamente já disponibilizando o áudio. Para a opção "Mensagem" o plugin irá apenas acessar o sistema de arquivo para obter conteúdo necessário para a animação.

A arquitetura do Avatar utiliza atualmente dois componentes principais, o framework FIVE [7] e o Avatar Vocálico. O FIVE foi desenvolvido com a intenção de auxiliar o desenvolvimento de aplicações com interface de voz. Atualmente o FIVE utiliza o VL Synthesizer engine para realizar a síntese de voz. O Avatar Vocálico é responsável pela geração dos visemas, que são uma sequência de imagens que o avatar irá exibir na animação. A Figura 2 mostra o processo realizado.

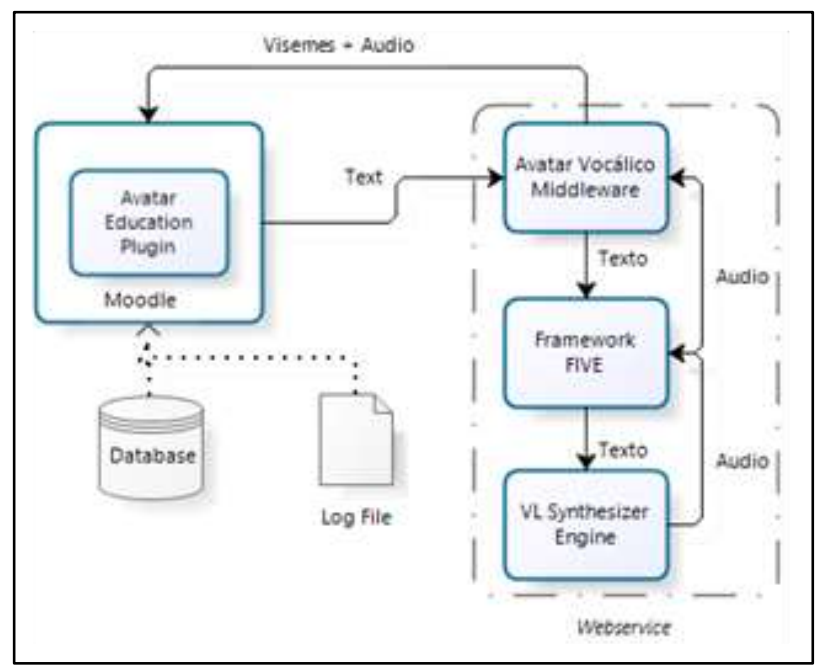

Figura 2: Arquitetura do Avatar. Maciel (2014).

O fluxo do processo conforme demonstra a Figura 2 parte do plugin localizado no ambiente Moodle que irá consultar o banco de dados para obter o número de novas atividades adicionadas, novos materiais, atividades pendentes ou mensagens deixadas pelo tutor, em seguida este resultado será comparado com o arquivo de log, que possui o resultado da última reprodução. Havendo novidades será o texto gerado será encaminhado para o Avatar Vocálico que irá gerar os visemas e solicitar o áudio ao framework FIVE.

\subsection{Arquiteturas Orientadas a Serviço}

Arquiteturas orientadas a serviços, Service Oriented Architecture (SOA), surgiram através da demanda de padronização da infraestrutura de TI visando a integração entre sistemas de plataformas diferentes bem como a manutenibilidade desses.[8]

Um exemplo de utilização de SOA é apresentado em Upadhyaya et al. [10] em que foram extraídas várias tarefas similares presentes nos sistemas, tais como a realização de um login. No estudo foram verificadas 21 aplicações de 4 domínios diferentes. As tarefas foram agrupadas em 2 categorias, precisa ser reusável ou precisa realizar uma funcionalidade específica do domínio.

Em OASIS[8] é definido que arquiteturas SOA são um "paradigma para organização e utilização de competências distribuídas que estão sob controle de diferentes domínios proprietários."

O paradigma SOA é fundamentado por três conceitos: visibilidade, interação e efeitos OASIS [8]. Visibilidade se refere a como um serviço está disponibilizado. A interação provê a forma de troca de mensagens, a ação com que os consumidores utilizarão os serviços. $\mathrm{O}$ efeito é o resultado da interação, pois ao consumir um determinado serviço se terá um efeito no mundo real. Em arquiteturas SOA os serviços são oferecidos de forma a abstrair os detalhes de implementação.

\subsubsection{Web Services}

Devido as vantagens e oportunidades de negócios oferecidos pelo paradigma de arquiteturas orientadas a serviços surgiram os web services [13], que se tratam de aplicações disponibilizadas no ambiente web. Estes serviços são acessados através do protocolo HTTP.

A utilização de web services também facilita a integração entre sistemas, bem como a comunicação com outros serviços. A arquitetura de um web service é fundamentada em dois princípios: especificação e descoberta [13]. A especificação detalha o serviço, facilitando a interação do requisitante com o serviço. A descoberta irá possibilitar o uso do serviço pelo requisitante.

Existem dois tipos de serviços web: os que utilizam o protocolo SOAP e os serviços REST. Os serviços SOAP (Simple Object Access Protocol) são descritos através do WSDL (Web Service Description Language), que é um contrato de uso daquele serviço. As mensagens SOAP 
trafegadas utilizam o formato XML e são transmitidas pelo protocolo HTTP [11]. Os sistemas que utilizam este tipo de serviços precisam seguir as especificações disponibilizadas no WSDL. Devido a falta de flexibilidade dos serviços SOAP posteriormente em [14] foi o criado paradigma REST (Representational State Transfer), que visa diminuir a dependência da especificação imposta pelo WSDL.

O REST tem como ênfase a escalabilidade das aplicações, segurança e baixo acoplamento. Um serviço utilizando REST utiliza o protocolo HTTP e seus métodos: PUT, POST, GET, DELETE. Nesta comunicação o Client irá informar os parâmetros necessários para uma requisição utilizando o protocolo HTTP, que são o cabeçalho e o corpo da mensagem. No cabeçalho serão passados dados como o endereço do serviço (Host), o método a ser utilizado entre outros dados como o tipo da mensagem. No corpo será passada a mensagem para o serviço.

O Server após receber a mensagem irá responder seguindo o padrão HTTP que deve informar o tipo da resposta, se houve sucesso e o conteúdo. A Figura 3 mostra o fluxo de uma requisição utilizando REST.

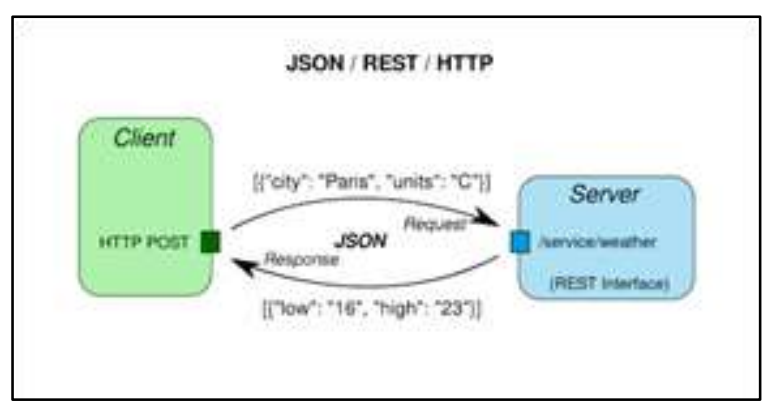

Figura 3: Fluxo de um Web Service utilizando REST.

No exemplo acima foi utilizado o formato JSON ( $\mathrm{Ja}$ vaScript Object Notation) para representar uma mensagem de requisição e de resposta. Também é possível fazer uso de outros formatos de mensagem como XML.

\section{Descrição da Proposta}

Em Upadhyaya et al. [10] foi realizado, no ambiente Moodle, um trabalho de refatoração de arquitetura propondo atender ao paradigma SOA. No estudo as atividades que possuíam similaridades, as atividades de autenticação de usuário, foram agrupadas e disponibilizadas em um serviço web. No Plugin Avatar de Educação foram analisadas as atividades realizadas de forma a separá-las e disponibilizadas em um serviço web chamado Avatar Service. A Tabela 1 mostra a distribuição dessas atividades com seus respectivos responsáveis.

\begin{tabular}{c|l|c}
\hline Ordem & \multicolumn{1}{|c}{ Atividade } & $\begin{array}{c}\text { Respon- } \\
\text { sável }\end{array}$ \\
\hline 1 & $\begin{array}{l}\text { Armazenamento das imagens do } \\
\text { Avatar para animação }\end{array}$ & Plugin \\
\hline 2 & Reprodução do áudio e animação. & Plugin \\
\hline 3 & $\begin{array}{l}\text { Consulta no banco de dados do } \\
\text { Moodle. }\end{array}$ & $\begin{array}{c}\text { API no } \\
\text { Moodle }\end{array}$ \\
\hline 4 & $\begin{array}{l}\text { Cadastro dos dados sobre Avatar } \\
\text { como o gênero e os tipos de noti- } \\
\text { ficações a serem reproduzidas. }\end{array}$ & $\begin{array}{c}\text { Avatar } \\
\text { Service }\end{array}$ \\
\hline 5 & $\begin{array}{l}\text { Construção de mensagem padrão } \\
\text { para notificações. }\end{array}$ & $\begin{array}{c}\text { Avatar } \\
\text { Service }\end{array}$ \\
\hline 6 & $\begin{array}{l}\text { Armazenamento do resultado } \\
\text { obtido pelas consultas realizadas } \\
\text { no banco de dados do Moodle. }\end{array}$ & $\begin{array}{c}\text { Avatar } \\
\text { Service }\end{array}$ \\
\hline 7 & $\begin{array}{l}\text { Cadastro de mensagens deixadas } \\
\text { pelos tutores. }\end{array}$ & $\begin{array}{c}\text { Avatar } \\
\text { Service }\end{array}$ \\
\hline 8 & $\begin{array}{l}\text { Comunicação com o Avatar Vocá- } \\
\text { lico. }\end{array}$ & $\begin{array}{c}\text { Avatar } \\
\text { Service }\end{array}$ \\
\hline
\end{tabular}

Tabela 1: Distribuição das atividades

Como é possível observar na Tabela 1 o plugin tornou-se mais simples, realizando atividades referentes basicamente a reprodução da animação do avatar. Isto favorece, inclusive, o processo de instalação em outra plataforma, além de facilitar sua manutenção.

A atividade de consulta no banco de dados do Moodle é necessária para verificar as novidades no AVA. Esta atividade foi atribuída a uma API desenvolvida no ambiente Moodle. O AVA oferece uma interface para comunicação com sistemas externos, esta funcionalidade é chamada de Web Service Externo, ela permite sistemas externos ao Moodle, como os web services, realizarem operações sobre o AVA, tais como a de criar um novo usuário no ambiente.

Apesar de existirem várias atividades padrão sobre o Moodle através dos Web Services Externos, foi preciso, para o Avatar Service, a criação de um Web Service Externo para realizar as consultas específicas de novas atividades, atividades pendentes e novos materiais deixados pelo tutor após o último login realizado. Este Web Service Externo foi descrito na arquitetura como sendo uma API do ambiente Moodle. A Figura 4 mostra a proposta da nova arquitetura.

Apesar de aumentar o caminho de requisição para o processo de síntese de voz quando comparado com a arquitetura anterior, a inclusão do Avatar Service neste processo possui alguns benefícios, tais como a customização da mensagem a ser reproduzida, o armazenamento dos dados de reproduções anterior ao invés de um arquivo de $\log$, a simplificação das atividades realizadas pelo plugin no ambiente Moodle. 


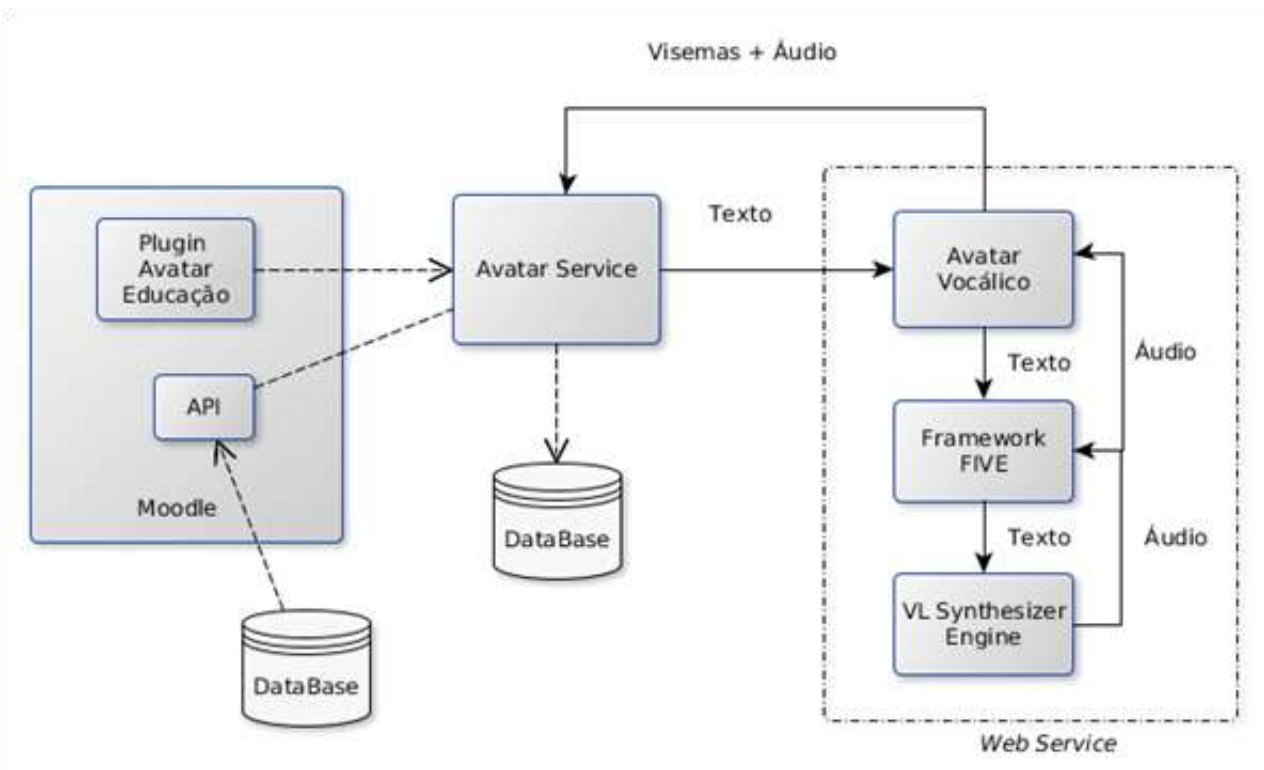

Figura 4: Proposta da nova arquitetura

O fluxo das atividades parte da interação do aluno com o plugin, que poderá acionar o botão "notificação" ou "mensagem". Quando selecionada a opção "notificação" haverá uma solicitação ao Avatar Service. O serviço irá obter os dados do ambiente Moodle através da API e irá comparar o resultado com sua base local, caso existam novidades será criada uma mensagem padrão e encaminhada ao Avatar Vocálico para a síntese de voz, do contrário o plugin irá reproduzir o último arquivo já reproduzido e disponível.

Para a opção de "mensagem" o processo de síntese de voz se fará após o tutor salvar uma mensagem para os alunos, automaticamente essa mensagem será encaminhada ao Avatar Service que irá se comunicar com o Avatar Vocálico. Após receber o conteúdo necessário para a animação o Avatar Service passará para o plugin armazenar no sistema de arquivos local.

\section{Desenvolvimento do Serviço}

O Avatar Service foi desenvolvido utilizando a plataforma Java devido a possibilidade de reusabilidade e portabilidade. $\mathrm{O}$ serviço foi estruturado como um projeto MAVEN para o gerenciamento das dependências e o ambiente de desenvolvimento escolhido foi o Eclipse. O Avatar Service por se tratar de uma aplicação web necessita de um servidor que realize sua devida disponibilização, para isto foi utilizado o Tomcat na versão 8.0 como o servidor da aplicação. O projeto ficou organizado conforme a seguinte distribuição de pacotes:

- service: pacote responsável por receber e responder as requisições recebidas pelo plugin do Avatar no Moodle;
- control: pacote que irá verificar se é necessário realizar o processo de síntese de voz;

- API: pacote que irá se comunicar com a API disponibilizada no ambiente Moodle;

- dao: pacote que possui métodos e classes necessárias para a comunicação com o banco de dados local;

- model: pacote que conterá as classes que representam o domínio do negócio, ou seja o gênero do avatar, curso, aluno solicitante, o tipo do conteúdo (notificação ou mensagem), entre outras informações.

Em Sommerville [5] é descrito que para se projetar uma arquitetura, tendo como requisito crítico a facilidade na manutenção, deve-se utilizar componentes de baixa granularidade e autocontidos, que possam ser prontamente alterados. Desta forma o Avatar Service foi desenvolvido como um serviço web do tipo REST, visando possuir flexibilidade para a necessidade de mudanças

Tanto o Avatar Service quanto o Plugin se comunicam através de mensagens utilizando a notação JSON. Quando o Avatar necessitar ser carregado na tela será enviado ao serviço uma mensagem solicitando as configurações de exibição como o gênero e qual o personagem. Quando o aluno acionar o botão "notificação" serão enviados os seguintes dados: a instituição, o id do aluno, o id do curso, o ambiente, o tipo da mensagem e o locutor (tipo de voz).

A resposta do Avatar Service possui 3 parâmetros: o nome para os arquivos .mp3 e o .vis (arquivo dos visemas), o conteúdo do arquivo $\mathrm{mp} 3$ codificado e o conteúdo do arquivo .vis. A Figura 7 mostra um exemplo de requisição e resposta com o Avatar Service. 


\section{//Requisição}

POST /AvatarService/service/provider/content HTTP/1.1

Host: localhost: 8080

Content-Type: application/json

Cache-control: no-cache

Body :

\{

"institutionId": "UPE_CARUARU",

"studentId":2,

"courseId":2,

"speaker": "cid",

"environment": "moodle",

"type": 1

\}

\section{//Resposta}

\{

"visemContent":"...",

"audioContent":"...",

"fileName":"vl_notifications_user2_course2"

\}

Figura 7 : Exemplo de requisição ao Avatar Service

Tanto o arquivo .vis quanto o .mp3 são codificados pelo Avatar Service em base64 e o plugin ao receber este conteúdo irá decodificar e salvar no sistema de arquivos local, de forma a facilitar o acesso ao conteúdo a ser reproduzido mesmo quando não houver comunicação com o Avatar Service.

Este Requisito do sistema ter a capacidade de funcionar de modo offline é fundamental para ambiente de ensino à distância, pois a instituição de ensino terá condições de prosseguir com o programa acadêmico e não atrapalhar/adiar o semestre letivo, ocasionando a desvalorização ou mau uso do plugin Avatar Educação.

\section{Resultados}

Para avaliar o desempenho do serviço foram realizados testes de carga em três cenários. O primeiro cenário simulando 35 alunos realizando chamada ao serviço simultaneamente, o segundo cenário com 45 usuários simultâneos e o terceiro com 55. A Tabela 2 mostra o resultado das simulações realizadas. A operação realizada por esses usuários virtuais foi referente a solicitação de notificações, pois desta forma possibilita tanto a comunicação com o Avatar Service quanto com o Avatar Vocálico e o framework FIVE para realização do processo de síntese de voz.

\begin{tabular}{l|l|l|l|l}
\hline $\begin{array}{c}\text { Usuár- } \\
\text { ios }\end{array}$ & $\begin{array}{c}\text { Tempo } \\
\text { Mínimo } \\
\text { (ms) }\end{array}$ & $\begin{array}{c}\text { Tempo } \\
\text { Máximo } \\
\text { (ms) }\end{array}$ & $\begin{array}{c}\text { Tempo } \\
\text { Médio } \\
\text { (ms) }\end{array}$ & $\begin{array}{l}\text { Desvio } \\
\text { Padrão }\end{array}$ \\
\hline 35 & 1556 & 3864 & 2790 & 678,88 \\
\hline 45 & 1606 & 4598 & 3263 & 858,69 \\
\hline 55 & 1650 & 6761 & 4363 & $\begin{array}{l}1441,9 \\
5\end{array}$ \\
\hline
\end{tabular}

Tabela 2: Tempos de resposta do Avatar Service.

Os testes de carga foram realizados no servidor. Assim nesses testes não se verificou um cenário utilizando uma infraestrutura de rede ou em um ambiente distribuído. Também não foram verificadas possíveis vulnerabilidades de segurança neste computador. Este servidor além de prover o serviço do Avatar também ficou responsável por manter instalado e em funcionamento o ambiente Moodle.

Os testes também foram realizados tomando como referência aproximadamente 60 usuários simultâneos com um tempo médio de cinco segundos para cada requisição. Esta requisição permite realizar todo o processo de síntese de voz e acesso aos sistemas externos ao Plugin do Avatar no ambiente Moodle. 


\section{Conclusões}

Após o desenvolvimento e testes sobre a nova arquitetura foi possível observar que o Avatar Service obteve resultados satisfatórios para atender a uma turma de até 55 alunos, conforme experimento demonstrado na seção 4 . Com isso, a refatoração da atual arquitetura extraindo algumas atividades do plugin para um serviço web possibilita vários benefícios como por exemplo a reutilização do mesmo para atender a outros ambientes.

A utilização da linguagem Java para o desenvolvimento do serviço se mostrou satisfatória para atender aos requisitos funcionais e não funcionais da arquitetura existente. Vale ressaltar que alguns componentes como o Avatar Vocálico também foi desenvolvido utilizando a mesma plataforma, favorecendo a facilidade na manutenção do ecossistema.

O trabalho realizado neste estudo possibilita a extensão com a realização de outros trabalhos, como a integração do Avatar em outros ambientes além do ambiente Moodle; a melhoria na geração das mensagens motivacionais de forma a aumentar a interação com o ambiente e a geração de modelos preditivos sobre diversas variáveis que podem ser extraídas como foi o trabalho desenvolvido em SANTANA et al. [9].

\section{Referências}

[1] Litto, Fredic M. e Formiga, Marcos. (2009) "Educação a distância: o estado da arte". Pearson Education do Brasil Ltd., Brasil.

[2]

HAGUENAUER, C.J. e MARTINS, F.N. Investigação sobre a Eficiência dos Ambientes Virtuais de Aprendizagem, com Foco na Percepção do Aluno. Revista Educaoline, Vol 2, no 1. Janeiro/abril de 2008.

[3] MOORE, M. "Three types of interaction". In: American Journal of Distance Education, v. 3, n. 2, 1-7, 1989. Disponível em: http://www.ajde.com/Contents/vol3 2.htm\#edito rial. Acesso em: 10 out. 2015.

[4] Maciel, A., Rodrigues, R., Carvalho, E. Desenvolvimento de um assistente virtual integrado ao moodle para suporte a aprendizagem online. Anais do Congresso Brasileiro de Informática na Educação, 2014.

[5] Sommerville, Ian. (2007) "Engenharia de software, $8^{\circ}$ edição". Pearson Addison Wesley., Brasil.
[6] Lee, K., Hauptmann, A. G. and Rudnicky, A. The Spoken Word. Byte, pages 225-232, July 1990.

[7] Desenvolvimento de uma ferramenta para a produção de mídias utilizando personagem animado com síntese de voz. 2012.

[8] OASIS - Reference Model: SOA Architecture V1.0 - 2007.

[9] SANTANA, Leandro C.; MACIEL, Alexandre MA; RODRIGUES, Rodrigo L. Avaliação do Perfil de Uso no Ambiente Moodle Utilizando Técnicas de Mineração de Dados. In: Anais do Simpósio Brasileiro de Informática na Educação. 2014. p. 269.

[10] UPADHYAYA, Bipin; ZOU, Ying; KHOMH, Foutse. An approach to extract RESTful services from web applications. International Journal of Business Process Integration and Management, v. 7, n. 3, p. 213-227, 2015.

[11] CURBERA, Francisco et al. Unraveling the Web services web: an introduction to SOAP, WSDL, and UDDI. IEEE Internet computing, v. 6, n. 2, p. 86, 2002.

[12] NEWCOMER, Eric; LOMOW, Greg. Understanding SOA with web services (independent technology guides). Addison-Wesley Professional, 2004.

[13] W3C WORKING GROUP et al. Web services architecture. 2004.

[14] FIELDING, Roy Thomas. Architectural styles and the design of network-based software architectures. 2000. Tese de Doutorado. University of California, Irvine.

[15] COSTA, Ferdinand Camara da et al. A utilização de ambientes virtuais de aprendizagem no ensino presencial: Estudo de caso na disciplina de um programa de mestrado. 2009.

[16] Censo da educação superior. 2014. Disponível em:

http://download.inep.gov.br/educacao superior/c en-

so superior/documentos/2015/notas sobre o ce nso da educacao superior 2014.pdf Acesso em 25 out. 2015. 